\title{
Effect of Facial Massage on Static Balance in Individuals with Temporomandibular Disorder - a Pilot Study
}

Yasmin El Hage, PT, MSc, ${ }^{1,2 *}$ Fabiano Politti, PT, PhD, ${ }^{1,2}$ Carolina Marciela Herpich, PT, MSc, ${ }^{1}$ Dowglas Fernando Magalhães de Souza, DMd, MSc, ${ }^{1}$ Cid André Fidelis de Paula Gomes, PT, MSc, ${ }^{1,4}$ Cesar Ferreira Amorim, EEc,PhD,${ }^{4}$ Tabajara de Oliveira Gonzalez, PT, PhD,,${ }^{1,2}$ Daniela Aparecida Biasotto-Gonzalez, PT, PhD ${ }^{1,2}$

\author{
${ }^{1}$ Postgraduate Program in Rehabilitation Sciences, Laboratório de Biodinâmica do Movimento Humano, Universidade \\ Nove de Julho (UNINOVE), São Paulo, ${ }^{2}$ Department of Physical Therapy, Universidade Nove de Julho (UNINOVE), \\ São Paulo, ${ }^{3}$ Master's Program in Physical Therapy, Universidade da Cidade de São Paulo (UNICID), São Paulo, \\ ${ }^{4}$ Postgraduate Program in Biophotonics Applied Helth Sciences, Universidade Nove de Julho (UNINOVE), São Paulo, Brazil
}

Introduction: The influence of the neuromuscular system on the cervical region and mastication is directly associated with mandibular movements and neck posture. Normal occlusal homeostasis depends on complex sensory feedback mechanisms of the periodontal ligament, temporomandibular joint and other structures of the stomatognathic system. This feedback serves as a regulatory mechanism that helps determine the force and nature of muscle contractions. Alterations in the muscles of mastication, neck muscles, and occlusal characteristics constitute causal factors of imbalances in the postural muscle chains, leading to alterations in the center of pressure (CoP) of the feet. Thus, therapies that seek occlusal reestablishment, such as muscle relaxation techniques, may lead to a restructuring of the global equilibrium of the neuromuscular system and an improvement in body posture.

Purpose: The aim of the present pilot study was to investigate the immediate effect of facial massage on the $\mathrm{CoP}$ in the anteroposterior $\left(\mathrm{CoP}_{\mathrm{AP}}\right)$ and mediolateral $\left(\mathrm{CoP}_{\mathrm{ML}}\right)$ directions in individuals with temporomandibular disorder (TMD).

Methods: Twenty individuals with a diagnosis of TMD based on the Research Diagnostic Criteria for Temporomandibular Disorders (RDC/TMD) were submitted to a facial massage technique. $\mathrm{CoP}_{\mathrm{AP}}$ and $\mathrm{CoP}_{\mathrm{ML}}$ were evaluated using a force plate. Evaluations were performed under two visual conditions (eyes open and eyes closed) prior to resting in dorsal decubitus (baseline), after 10 minutes of rest (premassage) and after the administration of the massage technique (postmassage).

Results: No significant differences were found regarding $\mathrm{CoP}_{\mathrm{AP}}$ velocity with eyes open or the following aspects under either visual condition (eyes open or closed): $\mathrm{CoP}_{\mathrm{ML}}$ velocity, RMS of
$\mathrm{CoP}_{\mathrm{AP}}, \mathrm{RMS}$ of $\mathrm{CoP}_{\mathrm{ML}}$, and sway area. The only significant difference was found for mean $\mathrm{CoP}_{\mathrm{AP}}$ velocity with eyes closed.

Conclusions: While the results of the present study demonstrate the reliability of the reproduction of the data, facial massage had no immediate influence on postural control in individuals with TMD.

KEY WORDS: temporomandibular joint disorder; postural balance; physical therapy modalities

\section{INTRODUCTION}

Temporomandibular disorder (TMD) is mainly a pathological condition of the muscles of mastication and postural muscles of the head and neck or combined pathological alterations of these muscles and the temporomandibular joint. Abnormalities in trigeminal afference and proprioception stemming from TMD can cause an imbalance of the entire postural muscle chain through a descending action, with a consequent effect on posture. ${ }^{(1)}$ Recent studies seem to demonstrate the role of trigeminal afference and alterations in the stomatognathic system with regard to proprioception, visual stabilization, and postural stability..$^{(2,3,4)}$

The literature describes numerous anatomic connections between the trigeminal system and nerve structures involving the maintenance of posture..$^{(2,5-12)}$ These interrelations demonstrate that information stemming from trigeminal afference may be linked to other types of afference involved in postural control. Perturbations in the stomatognathic system due to alterations in the muscles of mastication, periodontal ligament or temporomandibular joint could, therefore, lead to a perturbation in postural control due to the 
relationships among different neural nuclei in the brain stem. ${ }^{(13)}$

Massaging the muscles of mastication has proven to be effective in the treatment of TMD. ${ }^{(14-18)}$ In general, massage results in a reduction in pain, an increase in joint mobility, the elimination of adherences among muscle fibers, an increase in local circulation, and overall relaxation. ${ }^{(19)}$ Massage therapy leads to an increase in the range of motion of the mandible, a reduction in pain, and a reduction in the electromyographic activity of the muscles. ${ }^{(18,20)}$ However, little is known regarding the effects of massage on postural control.

While there is no evidence that massage therapy produces a movement toward the myocentric positioning of the jaw, it is believed that different conditions, such as continuous or intermittent low muscle activity, may be involved in this mechanism. ${ }^{(21,22,23)}$ Thus, it is possible that muscle relaxation may lead to balance between the right and left sides, with the consequent centric position of the mandible.

Studies have shown that the myocentric position of the mandible improves postural balance. ${ }^{(1,24)}$ Therefore, as TMD can alter the activity of the muscles of mastication, it is suggested that therapies aimed at the relaxation of these muscles, such as massage, could alter afference from receptors of the stomatognathic system, thereby affecting postural control. Based on this hypothesis, the aim of the present pilot study was to determine the immediate effect of facial massage on static balance in individuals with TMD.

\section{METHODS}

\section{Study Design}

A pilot study was carried out employing a $1 \times 3$ factorial design (treatment $\mathrm{X}$ time). The independent variables were treatment ( 1 intervention: facial massage) and time ( 3 conditions: baseline, premassage, and postmassage). The dependent variables resulted from the center of pressure (CoP): displacement amplitude (RMS), average sway velocity, and sway area.

\section{Sample}

The volunteers were recruited and screened based on pre-established inclusion criteria: either gender, age between 16 and 36 years, diagnosis of TMD, mandibular deviation and/or deflection, and complete dentition (except third molars). The following were the exclusion criteria: crossbite, mandibular prognathism or retrognathism, use of dentures, current orthodontic or physiotherapeutic treatment, neurological disorder, or use of orthopedic insoles. Twenty-three individuals met the eligibility criteria and agreed to participate in the study. Three did not complete the evaluations and were excluded from the analysis. Thus, 20 individuals with TMD (3 men and 17 women; mean age: $26.95 \pm 6.33$; mean height: $1.67 \pm 0.06$; mean weight: $67.75 \pm 8.88$ ) completed the study. Following the definition of the sample, the individuals were submitted to stabilometric evaluations and massage therapy. This study was carried out in compliance with the norms that regulate research involving human subjects contained in Resolution 196/97 of the Brazilian National Health Council. All procedures received approval from the Human Research Ethics Committee of the Universidade Nove de Julho (Brazil). The methodology employed in the present study is part of a protocol registered with the Brazilian Registry of Clinical Trials (RBR9x8ssz).

\section{Evaluations}

A trained examiner evaluated the individuals with regard to the presence of TMD using the Research Diagnostic Criteria for Temporomandibular Disorders (RDC/TMD), ${ }^{(25)}$ which is a biaxial diagnostic tool composed of a detailed physical evaluation of the mouth opening pattern, joint noises, and the extension of mandibular movements. The RDC/TMD questionnaire is made up of 31 items addressing general health, oral health, history of facial pain, mouth opening limitation, joint noises, habits, bite, ringing in the ears, general health status, joint problems, headache, current behavior, and socioeconomic profile. The diagnosis is established based on the combined results of the two axes. The RDC-TMD has been widely used in the literature(26-28) with the aim of enhancing the level of consistency among studies addressing TMD.

\section{Static Balance}

CoP measurements were determined using a portable force plate (BIOMEC 4000 v.1.1; EMG System do Brasil, São Jose dos Campos, Brazil) consisting of four load cells and a sampling frequency of 100 $\mathrm{Hz}$, connected to a computer. The participants were evaluated in a relaxed standing position with unrestricted width of the foot base, heels in alignment, arms alongside the body, and gaze fixed on a circular target ( $5 \mathrm{~cm}$ in diameter) at the height of the glabellum positioned at a distance of two meters. Two 60-second readings were performed under two visual conditions: eyes open and eyes closed.

To calculate the oscillation in the CoP, values referring to anteroposterior sway $\left(\mathrm{CoP}_{\mathrm{AP}}\right)$ and mediolateral sway $\left(\mathrm{CoP}_{\mathrm{ML}}\right)$ were filtered with a 4 th order Butterworth filter with a low-pass frequency of 10 Hz. The following variables were then calculated: displacement amplitude by the root mean square (RMS) of the signal, average sway velocity by the total displacement of the CoP divided by time ${ }^{(29)}$ and sway area (area of CoP displacement). ${ }^{(30)}$ All processing and extraction of the variables of the signals obtained from the force plate were performed using 
the MATLAB program, version 7.1 (The MathWorks Inc., Natick, MA).

\section{Intervention}

Between the first and second stabilometric readings (baseline and premassage), the participants remained at rest, lying comfortably in the supine position, with knees bent, on a cot for 10 minutes. Following the second reading (premassage), the same position was used for the massage therapy. The massage technique proposed by Biasotto-Gonzalez and Bérzin ${ }^{(18)}$ was employed. The total procedure lasted 20 minutes, and consisted of synchronized kneading and sliding movements with a medium degree of pressure on the anterior temporal, masseter, and suprahyoid muscles, performed by the same trained therapist positioned cranially to the subject. Moisturizing lotion was used during the massage to reduce friction. The protocol was applied bilaterally in a simultaneous fashion in two repeated steps. Step 1 consisted of surface sliding movements in a clockwise circular motion over the anterior temporal muscle. Step 2 consisted of kneading movements in the cranial-caudal direction over the masseter muscle. The return to the initial position involved continuous sliding in the opposite direction passing over the suprahyoid muscles.

\section{Statistical Analysis}

The intraclass correlation coefficient (ICC) was used to determine the intrasession reliability of the values obtained between two collections (test/retest) at the baseline and premassage evaluations. ICC values greater than 0.80 indicated excellent reproducibility, values between 0.80 and 0.60 denoted good reproducibility, and values less than 0.60 denoted poor reproducibility. ${ }^{(31)}$ Regarding the stabilometric variables, the Shapiro-Wilk test demonstrated that the data exhibited asymmetric distribution. Thus, Friedman's repeated-measure analysis of variance and Dunn's post hoc test were used for comparisons of the values obtained at the three evaluation times (baseline, premassage, and postmassage). The level of significance for all statistical tests was set to $5 \%$ $(p<0.05)$. All analyses were performed using the Statistical Package for the Social Sciences (SPSS 20.0, SPSS Inc., Chicago, IL).

\section{RESULTS}

\section{Intra-session Reliability}

Table 1 displays the ICCs for the values corresponding to body sway on two tests per individual. Good to excellent reliability was generally demonstrated for sway area and velocity under both visual conditions ( 0.71 to 0.97$)$. However, poor
TABLE 1. ICC for Two Trials: Anteroposterior (AP) and Mediolateral (ML) Oscillations with Eyes Open (EO) and Closed (EC)

\begin{tabular}{lcccccc}
\hline & & \multicolumn{4}{c}{ Velocity $(\mathrm{cm} / \mathrm{s})$} & \multicolumn{2}{c}{ RMS (cm) } \\
& & $\begin{array}{c}\text { Area } \\
\left(\mathrm{cm}^{2}\right)\end{array}$ & CoPAP & CoPML & CoPAP & CoPML \\
\hline \multirow{3}{*}{ Baseline } & EO & 0.72 & 0.85 & 0.93 & 0.49 & 0.40 \\
& EC & 0.71 & 0.96 & 0.90 & 0.71 & 0.73 \\
& EO & 0.95 & 0.97 & 0.84 & 0.90 & 0.88 \\
& EC & 0.77 & 0.97 & 0.80 & 0.86 & 0.80 \\
\hline
\end{tabular}

reproducibility was found on the baseline evaluation for RMS with eyes open ( 0.40 to 0.49$)$, with good to excellent reliability regarding the other variables in this same evaluation (0.71 to 0.90$)$.

\section{Analysis of CoP Variables}

In the analysis of body sway prior to rest (baseline), after 10 minutes of rest (premassage) and after massage therapy (postmassage) under two visual conditions (eyes open and eyes closed), the only significant difference was found for average $\mathrm{CoP}_{\mathrm{AP}}$ velocity with eyes closed (Table 2 ). No significant differences were found regarding $\mathrm{CoP}_{\mathrm{AP}}$ velocity with eyes open or regarding the following aspects under either visual condition (eyes open or closed): $\mathrm{CoP}_{\mathrm{ML}}$ velocity, RMS of $\mathrm{CoP}_{\mathrm{AP}}$, RMS of $\mathrm{CoP}_{\mathrm{ML}}$, and sway area (Table 2).

\section{DISCUSSION \& CONCLUSION}

The findings of the present study are supported by the adequate reliability in the reproduction of the data. Thus, although a significant difference was found in $\mathrm{CoP}_{\mathrm{AP}}$ velocity with eyes closed, the results demonstrate that one session of facial massage therapy does not exert an immediate influence on postural control (Table 2).

Although the massage protocol employed in the present study did not affect static postural control, which is similar to findings reported in previous studies involving the manipulation of different occlusal interferences, ${ }^{(5,13,32)}$ the results should be considered inconclusive. Significant changes in $\mathrm{CoP}$ have been described following different afferent stimuli through changes in occlusal positioning $(1,2,3,24)$ and the use of mandibular mobilization. ${ }^{(33)}$ The divergent findings may indicate that the effects of massage therapy are limited to local action, with a reduction in tension/pain ${ }^{(15)}$ and electromyographic activity ${ }^{(18)}$ in the muscles of mastication, as well as improvements in the signs and symptoms of TMD. ${ }^{(15,17,18)}$ 
TABle 2. Mean and Standard Deviation and Friedman's ANOVA Test (X2) Results of the Comparison Among CoP Data Recorded in Three Massage Moments (Baseline, Pre- and Postmassage) in Two Visual Conditions - Eyes Open (EO) and Eyes Close (EC)

\begin{tabular}{|c|c|c|c|c|c|c|}
\hline & & Baseline & Premassage & Postmassage & $\chi^{2}$ & $p$ value \\
\hline \multirow{2}{*}{ Area $\left(\mathrm{cm}^{2}\right)$} & EO & $1.58 \pm 0.99$ & $1.60 \pm 1.21$ & $1.75 \pm 1.24$ & 0.10 & 0.95 \\
\hline & $\mathrm{EC}$ & $1.93 \pm 1.24$ & $2.23 \pm 1.73$ & $2.32 \pm 1.41$ & 0.90 & 0.63 \\
\hline \multirow{2}{*}{ RMS CoP ${ }_{\mathrm{AP}}(\mathrm{cm})$} & $\mathrm{EO}$ & $0.42 \pm 0.17$ & $0.41 \pm 0.16$ & $0,38 \pm 0,13$ & 0.53 & 0.76 \\
\hline & EC & $0.42 \pm 0.12$ & $0.44 \pm 0.13$ & $0.51 \pm 0.19$ & 5.20 & 0.07 \\
\hline \multirow{2}{*}{$\mathrm{RMS} \mathrm{CoP}_{\mathrm{ML}}(\mathrm{cm})$} & EO & $0.20 \pm 0.07$ & $0.19 \pm 0.06$ & $0.23 \pm 0.08$ & 4.52 & 0.10 \\
\hline & $\mathrm{EC}$ & $0.24 \pm 0.12$ & $0.25 \pm 0.12$ & $0.24 \pm 0.09$ & 0.98 & 0.61 \\
\hline \multirow{2}{*}{ Velocity $\mathrm{CoP}_{\mathrm{AP}}(\mathrm{cm} / \mathrm{s})$} & EO & $5.33 \pm 1.55$ & $5.13 \pm 1.67$ & $5.33 \pm 1.83$ & 0.40 & 0.81 \\
\hline & $\mathrm{EC}$ & $4.99 \pm 1.81^{\mathrm{b}}$ & $4.90 \pm 1.49$ & $5.27 \pm 1.56^{\mathrm{b}}$ & 6.25 & $0.04^{\mathrm{a}}$ \\
\hline \multirow{2}{*}{ Velocity $\mathrm{CoP}_{\mathrm{ML}}(\mathrm{cm} / \mathrm{s})$} & $\mathrm{EO}$ & $0.79 \pm 0.67$ & $0.69 \pm 0.49$ & $0.74 \pm 0.35$ & 2.67 & 0.75 \\
\hline & $\mathrm{EC}$ & $0.84 \pm 0.68$ & $0.70 \pm 0.46$ & $0.74 \pm 0.39$ & 0.40 & 0.81 \\
\hline
\end{tabular}

aStatistically significant differences ( $p<.05$; Friedman's ANOVA test).

bStatistically significant differences in pairwise comparison (Dunn's Test).

$\mathrm{CoP}_{\mathrm{AP}}=$ center of pressure anteroposterior direction; $\mathrm{CoP}_{\mathrm{ML}}=$ center of pressure mediolateral direction.

Changes in electromyographic activity in the muscles of mastication and muscles of the neck have been reported following an immediate change in mandibular position. ${ }^{(34)}$ Trigeminal inputs from periodontal receptors (due to changes in the location of occlusal contacts), temporomandibular joint receptors (due to rotation and/or translation of the condyles), and muscle receptors (due to changes in mandibular position) may play a role in the modulation of the motor neuron pools of the cervical muscles with the change in the position of the mandible. ${ }^{(35)}$ Moreover, studies suggest that afferent activity from the orofacial region should be considered a significant input to head and neck motor control mechanisms. ${ }^{(36)}$ This may be due to the relationship between the descending tract of the trigeminal nerve and the upper cervical dorsal roots. (37) Neurons of the three divisions of cranial nerve $\mathrm{V}$ and cranial nerves VII, IX, and X share the same pools with neurons from the upper cervical spine. ${ }^{(37)}$

The maintenance of balance is a complex process that involves neural, muscular, skeletal, visual, vestibular, and other components. Information from the sensory systems is used by the postural control system for the internal representation of the spatial configuration of the body in a process that requires the activation of superior centers of the nervous system, especially the brain stem and cerebellum. ${ }^{(38)}$ Although the mechanisms used for the representation of body status are not yet fully clarified, the postural control system is known to recognize when sensory information is precise and attributes greater importance to such information. ${ }^{(39)}$

Despite the fact that massage is widely employed in clinical practice, the number of sessions, duration of the technique, and the use of only one massage therapy technique could be considered limitations of the present study. Thus, further investigation is needed to completely refute the hypothesis that massage therapy applied to facial muscles exerts no influence on postural control in individuals with TMD. However, the findings of this pilot study can be used as the basis of future investigations, including as a source for the calculation of sample size.

\section{ACKNOWLEDGMENTS}

Brazilian Registry of Clinical Trials (RBR9x8ssz).

\section{CONFLICT OF INTEREST NOTIFICATION}

The authors declare there are no conflicts of interest.

\section{COPYRIGHT}

Published under the CreativeCommons AttributionNonCommercial-NoDerivs 3.0 License. 


\section{REFERENCES}

1. Bracco P, Deregibus A, Piscetta R. Effects of different jaw relations on postural stability in human subjects. Neuroscience Letters. 2004;356(3):228-230.

2. Gangloff P, Louis JP, Perrin PP. Dental occlusion modifies gaze and posture stabilization in human subjects. Neuroscience Letters. 2000;293(3):203-206.

3. Gangloff P, Perrin PP. Unilateral trigeminal anaesthesia modifies postural control in human subjects. Neuroscience Letters. 2002;330(2):179-182.

4. Milani RS, De Pierre DD, Lapeyre L, Pourreyron L. Relationship between dental occlusion and posture. Cranio. 2000;18(2):127-134.

5. Michelotti A, Buonocore G, Farella M, Pellegrino G, Piergentili C, Altobelli S, et al. Postural stability and unilateral posterior crossbite: is there a relationship? Neuroscience Letters. 2006;392(1-2):140-144.

6. Dauvergne C, Nadiaye A, Buisseret-Delmas C, Buisseret P, Vanderworf F, Pinganaud G. Projections from the superior colliculus to the trigeminal system and facial nucleus in the rat. J Comp Neurol. 2004;478(3):233-247.

7. Kibana Y, Ishijima T, Hirai T. Occlusal support and head posture. J Oral Rehabil. 2002;29(1):58-63.

8. Pinganaud G, Bourcier F, Buisseret-Delmas C, Buisseret P. Primary trigeminal afferents to the vestibular nuclei in the rat: existence of a collateral projection to the vestibule-cerebellum. Neuroscience Letters. 1999;264(1-3):133-136.

9. Huggare J, Harkness E. Association between head posture and dental occlusion [abstract]. J Dental Res. 1993;72(1Suppl):255.

10. Pompeiarto M, Palacios JM, Mengodb G. Distribution and cellular localization of mRNA coding for 5-HT1A receptor in the rat brain: correlation with receptor binding. Neuroscience Letters. 1992;12(2):440-453.

11. Marfurt CF, Rajchert DM. Trigeminal primary afferent projections to "non-trigeminal" areas of the rat central nervous system. J Comp Neurol. 1991;303(3):498-511.

12. Buisseret-Delmas C, Compoint C, Delfini C, Buisseret P. Organisation of reciprocal connections between trigeminal and vestibular nuclei in the rat. J Comp Neurol. 1999;409(1):153-168.

13. Tardieu C, Dumitrescu M, Giraudeau A, Blanc J, Cheynet F, Borel L. Dental occlusion and postural control in adults. Neuroscience Letters. 2009;450(2):221-224.

14. Kalamir A, Pollard H, Vitiello AL, Bonello R. Manual therapy for temporomandibular disorders: a review of the literature. $J$ Bodywork Movement Therapies. 2007;11(1):84-90.

15. Albertin A, Kerpers II, Amorim CF, Costa RV, Corrêa JCF, Oliveira CS. The effect of manual therapy on masseter muscle pain and spasm. Eletromyogr Clin Neurophysiol. 2010;50(2):107-112.

16. Medlicott MS, Harris SR. A sistematic review of the efectiveness of exercise, manual therapy, electrotherapy, relaxation, training, and biofeedback in the management of temporomandibular disorder. Phys Ther. 2006;86(7):955-973.

17. Capellini VK, de Souza GS, de Faria CR. Massage therapy in the management of myogenic TMD: a pilot study. J Appl Oral Sci. 2006;14(1):21-26.
18. Biasotto-Gonzalez DA, Bérzin F. Electromyographic study of patients with masticatory muscles disorders, physiotherapeutic treatment (massage). Brazilian J Oral Sci. 2004;3(10):516-521.

19. Moyer CA, Rounds J, Hannum JW. A meta-analysis of massage therapy research. Psychol Bull. 2004;130(1):3-18.

20. Katsoulis J, Richter M. [Efficacité de la physiothérapie spécialisée sur les Sadam musculaires] [article in French]. Rev Stomatol Chir Maxillofac. 2008;109(1):9-14.

21. Miles TS. Postural control of the human mandible. Archives of Oral Biology. 2007;52(4):347-352.

22. Peck CC, Sooch AS, Hannam AG. Forces resisting jaw displacement in relaxed humans: a predominately viscous phenomena. J Oral Rehabil. 2002;29(2):151-160.

23. Jaberzadeh S, Brodin P, Flavel SC, O'Dwyer NJ, Nordstrom MA, Miles TS. Pulsatile control of the human masticatory muscles. J Physiol. 2003;547:613-620.

24. Fujino S, Takahashi T, Ueno T. Influence of voluntary teeth clenching on the stabilization of postural stance disturbed by electrical stimulation of unilateral lower limb. Gait \& Posture. 2010;31(1):122-125.

25. Dworkin SF, Huggins K, Wilson L, Mancl L, Turner J, Massoth $\mathrm{D}$, et al. A randomized clinical trial using research diagnostic criteria for temporomandibular disorders-axis II to target clinic cases for a tailored self-care TMD treatment program. J Orofac Pain. 2002;16(1):48-63.

26. De Felício CM, Sidequersky FV, Tartaglia GM, Sforza C. Electromyographic standard- ized indices in healthy Brazilian young adults and data reproducibility. J Oral Rehabil. 2009;36(8):577-558.

27. Tartaglia GM, Rodrigues da Silva MA, Bottini S, Sforza C, Ferrario VF. Masticatory muscle activity during maximum voluntary clench in different research diagnostic criteria for temporomandibular disorders (RDC/TMD) groups. Manual Therapy. 2008;13(5):434-440.

28. Epker J, Gatchel R, Ellis IE. A model for predicting chronic TMD: practical application in clinical settings. $J$ Am Dental Assoc. 1999; 130:470-475.

29. Doyle TL, Newton RU, Burnett AF. Reliability of traditional and fractal dimension measures of quiet stance center of pressure in young, healthy people. Arch Phys Med Rehabil. 2005;86(10):2034-2040.

.30. Oliveira LF, Simpson DM, Nadal J. Calculation of area of stabilimetric signals using principal component analysis. Physiol Meas. 1996;17(4):305-312.

31. Bartko JJ. The intraclass correlation coefficient as a measure of reliability. Psychol Rep. 1966;19(1):3-11.

32. Ferrario VF, Sforza C, Miani JR, D'addona A, Barbini E. Electromyographic activity of human masticatory muscles in normal young people: statistical evaluation of reference values for clinical applications. J Oral Rehabil. 1993;20(3):271-280.

33. Amaral AP, Politti F, Hage YE, Arruda EC, Amorin CF, Biasotto-Gonzalez DA. Immediate effect of nonspecific mandibular mobilization on postural control in subjects with temporomandibular disorder: a single-blind, randomized, controlled clinical trial. Revista Brasileira de Fisioterapia.2013;17(2).

34. Ceneviz C, Mehta NR, Forgione A, Sands MJ, Abdallah EF, Lobo SL, Mavroudi S. The immediate effect of changing mandibular position on the EMG activity of the masseter, 
temporalis, sternocleidomastoid, and trapezius muscles. $J$ Craniomandibular Pract. 2006;24(4):237-244.

35. Miralles R, Moya H, Ravera MJ, Santander H, Zuniga C, Carvaial $R$, et al. Increase of the vertical occlusal dimension by means of a removable orthodontic appliance and its effect on craniocervical relationship and position of the cervical spine in children. J Craniomandibular Pract.1992;15:221-228.

36. Eriksson PO, Zafar H, Nordh E. Concomitant mandibular and head-neck movements during jaw opening-closing in man. $J$ Oral Rehabil. 1998;25(11):859-870.

37. Miralles R, Doods C, Manns A, Palazzi C, Jaramillo C, Quezada V, et al. Vertical dimention. Part 2: the changes in electrical activity of the cervical muscles upon varying the vertical dimension. Cranio. 2002;20(1):39-47.
38. Morasso PG, Schieppati M. Can muscle stiffness alone stabilize upright standing? J Neurophysiol. 1999;82(3):1622-1626.

39. Shumway-Cook A, Woollacott MH. Motor Control: Theory and Practical Applications, 2nd edition. Philadelphia, PA: Lippincott Williams \& Wilkins Inc; 2003.

Corresponding author: Yasman El Hage, PT, MSC, Postgraduate Program in Rehabilitation Sciences and Department of Physical Therapy, Universidade Nove de Julho (UNINOVE), Laboratório de Biodinâmica do Movimento Humano, Universidade Nove de Julho (UNINOVE), Av. Dr. Adolfo Pinto, 109 Água Branca, São Paulo 05001-100, SP, Brazil

E-mail: hage.fisio@gmail.com 